


\title{
(sntideidungen
}

\author{
bes \\ $\mathfrak{A} \mathfrak{e} \mathfrak{i} \mathfrak{d} \mathscr{G} \mathfrak{g} \mathfrak{e} \mathfrak{x} \mathfrak{i} \mathfrak{d} \mathfrak{t}$ \\ Serausgegeben \\ bon \\ Den Allitgliedern des Geridtshofes \\ und Der Reidganmalt/djaft.
}

(Entidjeibungen in $3^{i v i l}$ jadjent.

\section{Reue Folge.}

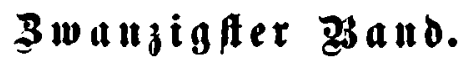

Drr gamąn Reilje liebz̨iglter Bant.

eripzig,

Berlag von Beit \& Eomp.

1909 


\title{
(Entjodoionngen
}

\author{
Des \\ $\mathfrak{A} \mathfrak{e} \mathfrak{i} \mathfrak{d} \mathfrak{g} \mathfrak{g} \mathfrak{e} \mathfrak{x} \mathfrak{i} \mathfrak{d} \mathfrak{t} \mathfrak{g}$ \\ in \\ Biviliaden.
}

Neue Folge.

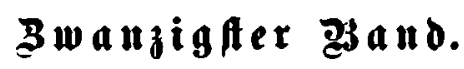

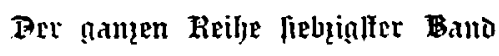

eripzig,

Berlag von Beit \& Comp.

1909 


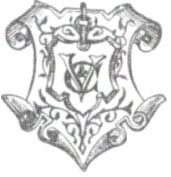

Irud bon Mrebacr \& Bittla in Leipsia 